Proceedings of the International School and Conference on Optics and Optical Materials, ISCOM07, Belgrade, Serbia, September 3-7, 2007

\title{
Dark Resonances in Quantum Optics
}

\author{
E. Arimondo
}

\author{
CNR-INFM and CNISM, Dipartimento di Fisica "E. Fermi" \\ Università di Pisa, Largo Pontecorvo 3, Pisa, Italy
}

The processes occurring in three-level systems are briefly reviewed. Coherent population trapping, bright resonances, electromagnetic-induced transparency, electromagnetic-induced transparency optical clock are discussed.

PACS numbers: 42.50.Gy

\section{Introduction}

Quantum control and quantum computation, representing two important directions of today research, rely on the precise modification of quantum mechanical systems not perturbed by an environment presence. This precise control was reached in different areas of physics using electromagnetic fields interacting with atoms or molecules in different matter phases. The simplest quantum mechanical system to be explored is a two-level atom, with lower and upper levels excited by a resonant or quasi-resonant electromagnetic field, as it occurs in nuclear or electronic magnetic resonance or in two-level laser spectroscopy. There the populations of the two levels or the coherence between the two levels are precisely modified by a control on the frequency, amplitude and interaction time of the electromagnetic field. The weak perturbation by the environment is realized, for instance, operating with low pressure or with cold/ultracold gases as produced by the combination of laser cooling and evaporative cooling techniques.

The next step in level complexity is the three-level systems, with three levels, to be denoted as $|0\rangle,|p\rangle,|c\rangle$ excited by two separate lasers, the first probe laser at frequency $\omega_{\mathrm{p}}$ driving the transition $|p\rangle \rightarrow|0\rangle$ and the coupling laser at frequency $\omega_{\mathrm{c}}$ driving the transition $|c\rangle \rightarrow|0\rangle$. The atom-laser interaction of a three-level system is based on the evolution of the population of the three levels, of the optical coherences between levels $|0\rangle,|p\rangle$ and $|0\rangle,|c\rangle$. In addition, the three-level system may be prepared by the applied lasers in a coherent superposition state, whence it supports one high order coherence between levels $|p\rangle$ and $|c\rangle$. The build up of that coherence by the applied lasers produces new processes and new phenomena, peculiar of the three-level configuration. Those processes and phenomena have stimulated a large attention by the laser spectroscopy and quantum optics 


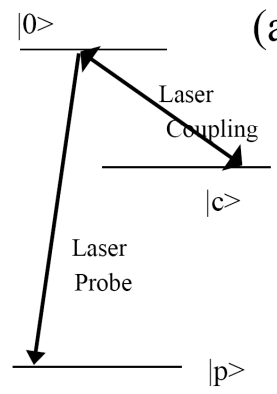

(a)

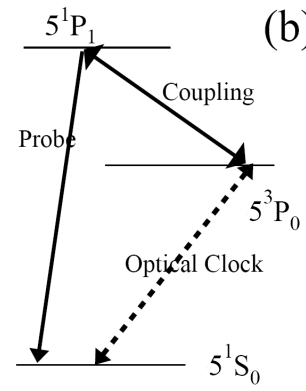

(b)

Fig. 1. (a) $\Lambda$ scheme with lower levels $|1\rangle$ and $|2\rangle$ and upper level $|3\rangle$. Applying the probe laser and the coupling laser a coherence is established between the lower levels, mediated by the upper one. (b) Three level ${ }^{88} \mathrm{Sr}$ configuration for an optical clock.

communities in the last thirty years, as presented in [1-4]. The three-level configuration denoted as $\Lambda$ with the levels $|p\rangle$ and $|c\rangle$ having lower energies and the upper level $|0\rangle$ separated by an energy splitting in the optical range, as schematically represented in Fig. 1a, constitutes the most interesting configuration. In fact, the time evolution of the high order coherence is not limited by the spontaneous emission processes from the upper level and takes place on a timescale much slower than the optical oscillation time. Therefore the phenomena of dark resonances (DR), coherent population trapping (CPT), electromagnetic-induced transparency (EIT) are greatly enhanced in the $\Lambda$ system.

When an atomic system is prepared in a coherent superposition state, very narrow resonances can be observed where the presence of the atomic coherence cancels, or reduces, absorption and greatly modifies the refractive index of the medium. The early study relevant to this situation includes measurement performed in Pisa and Rochester where terms such as dark resonance and CPT were introduced [5-7]. The continuely expanding interest in the topic is due not only to the fascinating physics involving quantum coherence but also to the fact that there are many potential applications with relevance both in possible implementation of new techniques and devices and in new scientific/research approaches to fundamental studies such as slowing of light, nonlinear optics, quantum information storage, frequency standards, precise magnetometers, and some more.

Section 2 of the present work introduces the basic concepts of the three-level phenomena, Sect. 3 discusses the proposed EIT application to the ${ }^{88} \mathrm{Sr}$ optical clock.

\section{Basic}

The three-level system considered here is shown in Fig. 1a. The energy separation between levels $|i\rangle$ and $|j\rangle$ denoted as $\hbar \omega_{i j}$. The common level $|0\rangle$ is coupled to both levels $|p\rangle$ and $|c\rangle$ through transitions induced by two applied classical laser fields, probe, and coupling lasers, respectively. It is supposed that either due to selection rules produced by a proper choice of the polarizations of 
the two laser fields or because of properly chosen frequency detunings, each laser field acts only on one transition. The laser detunings are $\delta_{\mathrm{p}}=\omega_{\mathrm{p}}-\omega_{0 \mathrm{p}}$ and $\delta_{\mathrm{c}}=\omega_{\mathrm{c}}-\omega_{0 \mathrm{c}}$, and the Raman detuning is $\Delta=\delta_{\mathrm{p}}-\delta_{\mathrm{c}}$. The Rabi frequencies $\Omega_{\mathrm{p}}$ and $\Omega_{\mathrm{c}}$ characterize the atom laser interaction. The main relaxation processes completing the three-level evolution are the spontaneous decay from upper level $|0\rangle$ with total rate $\Gamma$ and the decay rate $\gamma_{\mathrm{cp}}$ of the second order coherence between levels $|p\rangle$ and $|c\rangle$.

Very peculiar narrow features appear for the occupation of the $|0\rangle$ upper level when the two-photon condition is satisfied: i.e. around the two-photon Raman resonance condition $\Delta \approx 0$. Figure 2 , left part, shows numerical results for the line shape of the $W_{\text {abs }}$ absorption for the probe laser and that line shape is identical to line shape of the $|0\rangle$ level population. The coupling laser is fixed at its
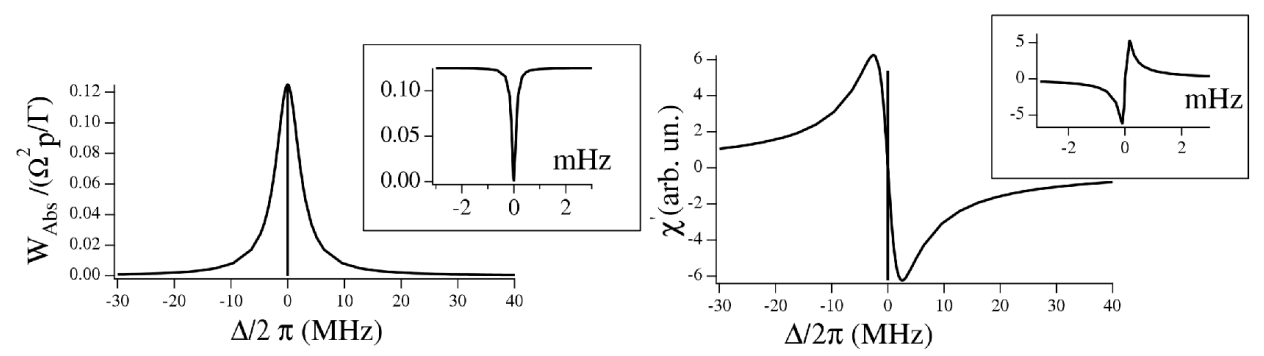

Fig. 2. Left - absorption rate of the $461 \mathrm{~nm}$ laser in the $\Lambda$ optical clock scheme of ${ }^{88} \mathrm{Sr}$ in the presence of the $1354 \mathrm{~nm}$ control laser. Right $-\chi^{\prime}$ susceptibility of the 461 $\mathrm{nm}$ laser in the ${ }^{88} \mathrm{Sr} \Lambda$ scheme. Laser parameters: $\delta_{\mathrm{c}}=0, \Omega_{\mathrm{c}}=10^{-6} \Gamma$. The insets show on an expanded scale the central EIT features.

resonance value $\delta_{\mathrm{c}}=0$, whereas the probe laser is swept around its optical resonance. When the two laser fields are scanned around the Raman resonance values, the probe absorption, and the population of the upper $|0\rangle$ level, increases following the Lorenztian profile of the absorption line. A maximum of both quantities is expected with both lasers in resonance. However, a strong decrease occurs in a narrow region around the Raman resonance, where the atoms remain distributed over the lower $|1\rangle$ and $|2\rangle$ levels, without occupation of the upper level. The population of the $\Lambda$ system is trapped in the lower states. The fluorescent emission from the upper level, which is proportional to the excited level occupation, presents a strong decrease at the Raman resonance value, that decrease denoted as dark line in the experimental observations of [5]. Away from the Raman resonance, the occupation of the three levels is determined by the optical pumping process. Because of the optical pumping redistribution among the three levels, the CPT results do not depend on the initial level populations. Similar results for the absorption and excited state population line shape are obtained with the coupling laser fixed at a value not corresponding to the optical resonance $\delta_{\mathrm{c}} \neq 0$ and scanning the frequency 
of the probe laser around the resonance Raman condition $\Delta=0$. CPT is again produced with a drastic decrease in the probe absorption and in the excited-state population. If we disregard $\gamma_{\mathrm{cp}}$ for a moment, the absorption rate vanishes exactly at $\Delta=0$, evidence of the EIT effect [1]. The occurrence of a zero in the absorption profile is clear from the connection of this problem to the Fano $[8,9]$ line shape, with an asymmetry produced by interference between different probe absorption processes. The EIT deep, narrow dip occurring in the absorption profile presents a width determined by $\gamma_{\mathrm{cp}}$ and the absorption rate $\tilde{\gamma}=\Omega_{\mathrm{c}}^{2} / \Gamma$.

A unitary transformation is very useful for understanding the CPT process. Let us analyze the simple case in which the two lower levels, $|p\rangle$ and $|c\rangle$ are degenerate and the two laser fields have the same frequency and phase. We may introduce the dark (or non-coupled) $\left|\Psi_{\mathrm{NC}}\right\rangle$ and bright (or coupled) states $\left|\Psi_{\mathrm{C}}\right\rangle$ to describe the atomic evolution between the lower levels

$$
\begin{aligned}
& \left|\Psi_{\mathrm{NC}}\right\rangle=\frac{\Omega_{\mathrm{c}}}{\sqrt{\Omega_{\mathrm{p}}^{2}+\Omega_{\mathrm{c}}^{2}}}|p\rangle-\frac{\Omega_{\mathrm{p}}}{\sqrt{\Omega_{\mathrm{p}}^{2}+\Omega_{\mathrm{c}}^{2}}}|c\rangle, \\
& \left|\Psi_{\mathrm{C}}\right\rangle=\frac{\Omega_{\mathrm{p}}}{\sqrt{\Omega_{\mathrm{p}}^{2}+\Omega_{\mathrm{c}}^{2}}}|p\rangle-\frac{\Omega_{\mathrm{c}}}{\sqrt{\Omega_{\mathrm{p}}^{2}+\Omega_{\mathrm{c}}^{2}}}|c\rangle .
\end{aligned}
$$

Because the transition matrix element between $|\mathrm{NC}\rangle$ and $|0\rangle$ vanishes, an atom in that state cannot absorb photon and be excited to $|0\rangle$. Atoms are pumped into $\left|\Psi_{\mathrm{NC}}\right\rangle$ after a few spontaneous emission cycles. Numerical results for the EIT narrow dip in the absorption profile are shown in the left part of Fig. 2 for the case of the $\Lambda$ configuration introduced in Sect. 3 .

The EIT process, characterized by a decreased absorption, is described by the absorption length $\zeta_{\mathrm{p}}^{\mathrm{EIT}}$, defining the exponential decay of the probe in the propagation

$$
\zeta_{\mathrm{p}}^{\mathrm{EIT}}=\zeta_{\mathrm{p}}\left(\frac{\Omega_{\mathrm{c}}^{2}}{2 \gamma_{\mathrm{cp}} \Gamma}+1\right)
$$

where $\zeta_{\mathrm{p}}$ denotes the probe Beer absorption length in the absence of coupling laser. Equation (3) predicts no probe attenuation for the case of $\gamma_{\mathrm{cp}}=0$.

The EIT narrow modification of the atomic response appears also in the real part of the optical susceptibility $\chi^{\prime}\left(\omega_{\mathrm{p}}\right)$ that determines the refractive index and the light propagation speed within the medium. The EIT modification of the refractive index, with a narrow and inverted dispersion line shape, is shown in the right part of Fig. 2. The group velocity $v_{\mathrm{g}}$ characterizing the propagation of a laser pulse through the EIT medium is given by

$$
v_{\mathrm{g}}=\frac{c}{1+\frac{\chi^{\prime}\left(\omega_{\mathrm{p}}\right)}{2}+\frac{\omega_{\mathrm{p}}}{2} \frac{\partial \chi^{\prime}\left(\omega_{\mathrm{p}}\right)}{\partial \omega_{\mathrm{p}}}}
$$

The EIT narrow dispersion peak produces slow light, propagating through the medium at very reduced speed.

\section{Sr optical clock}


In the field of optical frequency standards, alkaline earth atoms or ions are attractive candidates for new clock performances where resonance quality factors are expected to be better than microwave-based atomic clocks. A competitive proposal is to localize neutral cold atoms spatially in a Lamb-Dicke regime while the trapping potential is designed such that its presence does not influence the clock transition frequency. This scheme can be realized using a far-off-resonance dipole trap operating at a wavelength where the ground and the excited state of the clock transition experience exactly the same ac Stark shifts. A state with a scalar polarizability is preferred, to avoid the problem of the complex and sometimes uncontrolled light polarization inside an optical lattice. In [9] a scheme of an optical clock based on states of true scalar nature, namely $5 s^{2}{ }^{1} S_{0}-5 s 5 p{ }^{3} P_{0}$ of ${ }^{88} \mathrm{Sr}$ (with $I=0$ ) was proposed. A direct transition between these two states is of course completely forbidden, and the clock scheme is based instead on three-level quantum coherence established by two probe laser frequencies.

In the ${ }^{88} \mathrm{Sr} \Lambda$, since the ground state $\left(5 s^{2}{ }^{1} S_{0}\right)$ (state $\left.|p\rangle\right)$ and the first excited state $\left(5 s 5 p^{3} P_{0}\right)$ (state $\left.|c\rangle\right)$ of ${ }^{88} \mathrm{Sr}$ are characterized by a total angular momentum of $J=0$, electric and magnetic one-photon transition matrix elements between these two states vanish to any multipole order. The $5 s 5 p{ }^{1} P_{1}$ state (state $|0\rangle$ ) can be reached from $|p\rangle$ via E1 one-photon absorption using the probe laser at a wavelength of $461 \mathrm{~nm}$. One M1 photon from the coupling laser at $1354 \mathrm{~nm}$ is needed to resonantly couple $|c\rangle$ and $|0\rangle$. The latter decays primarily to $|p\rangle$ with $\Gamma_{0 \rightarrow \mathrm{p}}=2 \pi \times 32 \mathrm{MHz}$.

If the dressing laser is off, the Lorentzian profile connected with the $|p\rangle \rightarrow|0\rangle$ transition is recovered. Otherwise, the absorption rate vanishes at $\Delta=0$, as for EIT. For the narrow EIT dip occurring in the absorption profile, not only its position, but also its width is very insensitive with respect to fluctuations of $\delta_{\mathrm{c}}$. A variation of the dressing laser frequency by as much as $100 \mathrm{kHz}$ induces a relative change of the EIT width of less than $10^{-7}$. By measuring the absorption as a function of $\omega_{\mathrm{p}}-\omega_{\mathrm{c}}$, the clock frequency can be determined with high accuracy by tracking the position of the dip. Neither $\omega_{\mathrm{p}}$ nor $\omega_{\mathrm{c}}$ must be particularly stable, only the difference needs to be stabilized. For relatively large detuning $\left(\delta_{\mathrm{c}}=5 \Gamma\right)$, the signal amplitude is reduced and the peak disappears as soon as decoherence becomes appreciable.

In order to evaluate the potential accuracy of the proposed clock scheme, the ac Stark shifts due to nonresonant electric dipole coupling of the three levels to states inside and outside the three-level subspace should be considered. These ac shifts may be controlled by operating the clock at very low Rabi frequencies. However, this simple approach does not apply to EIT clocks where the laser intensity modifies also the optical pumping time to prepare atoms into coherent superposition characterizing the EIT response [4]. To overcome these limits, Refs. [10, 11] examined a scheme based on the interaction of the atomic clock transition with time separated Raman laser pulses, Fig. 3. Moreover, the detunings of the applied 


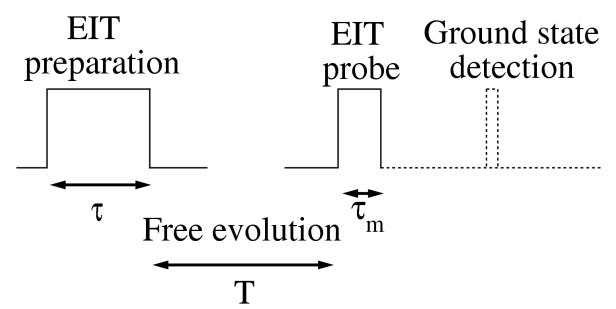

Fig. 3. The Ramsey pulse sequence probes the EIT resonance. EIT preparation takes place during the $\tau$ window. After a $T$ interval of free evolution, the EIT is interrogated during $\tau_{\mathrm{m}}$ with a later detection of the ground state occupation.

lasers change the phase evolution of the atomic wave function, so that a proper combination of "magic" laser detuning and pulse duration allows to compensate for the ac Stark shifts. In addition, the pulsed configuration produces a large contrast in the detected clock signal. This interrogation scheme represents an original mix of the Ramsey phase spectroscopy and high efficient population transfer under CPT acting as a quantum non-demolition measurement. This method performs the $10 \mathrm{mHz}$ accuracy expected for a "light-insensitive" lattice clock with Ramsey-EIT pulses to dilute fluctuations of the frequency resolution over probing time $\tau+T+\tau_{\mathrm{m}}$. The pulse approach remains valid for multi-photon excitation paths where dark states can be found as a clock transition.

\section{Acknowledgments}

This work was supported by the OLAQUI and EMALI European Projects and by MIUR-Italy PRIN Contracts. The Sr optical clock is based on a collaboration with C. Greene, R. Santra, Jun Ye and T. Zanon-Willette.

\section{References}

[1] S.E. Harris, Phys. Rev. Lett. 70, 552 (1993).

[2] E. Arimondo, Prog. Opt. 35, 257 (1996).

[3] M.D. Lukin, P.R. Hemmer, M.O. Scully, Adv. At., Mol., Opt. Phys. 42, 347 (2000).

[4] M. Fleischhauer, A. Imamoglu, J.P. Marangos, Rev. Mod. Phys. 77, 633 (2005).

[5] G. Alzetta, A. Gozzini, L. Moi, G. Orriols, Nuovo Cimento 36, 5 (1976).

[6] E. Arimondo, G. Orriols, Lett. Nuovo Cimento 17, 333 (1976).

[7] H.R. Gray, R.M. Whitley, C.R. Stroud, Opt. Lett. 3, 218 (1978).

[8] B. Lounis, C. Cohen-Tannoudji, J. Phys. II (France) 2, 579 (1992).

[9] R. Santra, E. Arimondo, T. Ido, C.H. Greene, Jun Ye, Phys. Rev. Lett. 94, $173002(2005)$.

[10] T. Zanon-Willette, A.D. Ludlow, S. Blatt, M.M. Boyd, E. Arimondo, Jun Ye, Phys. Rev. Lett. 97, 233001 (2006).

[11] T.H. Yoon, Phys. Rev. A 76, 013422 (2007). 